\title{
Study of Polyolefin Gel in Organic Solvents II. Structure and Morphology of Branched Low Density Polyethylene Gel in Organic Solvents
}

\author{
Hideomi MATSUDA, Ryoichiro KASHIWAGI, and Masaru OKABE* \\ Department of Fine Materials Engineering, Faculty of Textile Science, \\ Shinshu University, 3-15-1, Tokida, Ueda, Nagano 386, Japan \\ *Department of Chemical Process Engineering, Faculty of Engineering, \\ Ikutoku Technical University, 1030, Shimo-ogino, \\ Atsugi, Kanagawa 243-02, Japan
}

(Received September 11, 1987)

\begin{abstract}
Two kinds of branched low density polyethylene (BLDPE) gels were prepared by cooling a solution rapidly (a rapid cooling gel) and slowly (a slow cooling gel), and the morphology as well as the structure of the gels were studied. Moreover, gel-melting temperature $T_{\mathrm{m}}^{\mathrm{g}}$ was measured and its dependence on polymer concentration, molecular weight, and branching degree was also investigated. A range of weight-average molecular weight of samples used is from $8.7 \times 10^{4}$ to $17.5 \times 10^{4}$. For both rapid and slow cooling gels, $T_{\mathrm{m}}^{\mathrm{g}}$ increases with increasing polymer concentration as well as molecular weight, but decreases with increasing branching degree. The minimum polymer concentration $C^{*}$ for gel formation and the form of crystallites in gels depend on the cooling rate of a solution considerably, though the difference in $T_{\mathrm{m}}^{\mathrm{g}}$ between the rapid cooling gel and the slow cooling one for a same sample is little. The $C^{*}$ of both gels is much higher than the concentration $C_{0}$ at which polymer chains begin to overlap with each other in a solution, and gelation takes place through the molecular overlap as well as the crystallization from the solution.

KEY WORDS Sol-Gel Transition / Morphology / Structure of Gel / Thermo-Reversible Gel / Polyethylene / Branched Low Density Polyethylene / Gel-Melting Temperature / Spherulite /
\end{abstract}

Thermo-reversible sol-gel transition of polymers from solutions is a well-known phenomenon, ${ }^{1-8}$ and it is widely recognized that the gelation takes place as a consequence of three-dimensional network formation by chemical or physical linkage of flexible polymer chains. ${ }^{9,10}$ In a series of studies, ${ }^{11-18}$ we reported the thermo-reversible sol-gel transition of crystalline polymers such as branched low density polyethylene, linear low density polyethylene, linear high density polyethylene, and isotactic polypropylene in organic solvents, and showed that the sol-gel transition was one of the general properties of these crystalline polymers and the gelation took place through the crystallization of a polymer from a solution with cooling.

Recently, the mechanism of sol-gel transition has been investigated from the viewpoint of liquid-liquid phase separation or spinodal decomposition ${ }^{19-30}$ particularly for poorly crystallizable or noncrystallizable polymersolvent systems such as gelatin or agar in water, poly(vinyl alcohol) in water, isotactic polystyrene in decalin, atactic polystyrene in carbon disulfide, polyacrylonitrile in a mixed solvent of $\mathrm{N}, \mathrm{N}$-dimethylformamide-ethylene glycol, and poly(vinyl chloride) in $\gamma$-butyrolactone. That is, gelation takes place from phase separation into polymer-rich and polymer-poor regions and the network of gel is formed by the connectivity of polymer 
chains in the polymer-rich phase. On the other hand, Mandelkern and co-workers ${ }^{31}$ propose that, for copolymers or homopolymers of ethylene, gelation takes place as a consequence of crystallization, and liquidliquid phase separation or spinodal decomposition is not necessary for the gelation of these crystalline polymer solutions.

When a polymer solution converts to a gel, a three-dimensional network or crosslinking junction points should be formed in the gel. According to Takahashi and co-workers, ${ }^{1,32}$ a crystalline polymer with copolymeric characters such as chemically different units, stereoirregularities, or chain branching is conducive to gelation from a solution, and the junction points of the network of gel are made up of crystallites. This concept has been widely supported through experiments on X-ray diffraction and thermodynamics of gel-melting behavior of the crystallites. ${ }^{1,7}$ However, the morphology and structure of a polyolefin gel have not been satisfactorily clarified.

The purpose of this study is, therefore, to investigate the morphology as well as the structure of branched low density polyethylene (BLDPE) gel in organic solvents through observation of crystallites formed in a phase separation region and also in a gel region. For this purpose, two kinds of BLDPE gel were prepared by the following two different methods: One was prepared by cooling a BLDPE solution rapidly (a rapid cooling gel) and the other was prepared by cooling the solution very slowly (a slow cooling gel), and the difference of structure between these gels was investigated. Moreover, gel-melting temperatures of both gels were compared with each other, and the dependence of gel-melting temperature on polymer concentration, branching degree, and molecular weight was also investigated.

\section{EXPERIMENTAL}

\section{Materials}

Five samples of unfractionated BLDPE supplied by Showa Denko Co. were used. The physical properties of samples are tabulated in Table I. In BLDPE- $1-3$ or in BLDPE-4-5, weight-average molecular weights $\bar{M}_{w}$ determined by GPC are almost same but the numbers of methyl groups per 1000-carbon atoms $\mathrm{CH}_{3} / 1000 \mathrm{C}$, i.e., branching degree, are different.

Purification of the samples was carried out as follows: Pellets of the sample were dissolved completely in xylene at its boiling point under a reflux condenser and then precipitated into an excess of cooled methanol with stirring. After washing with methanol, the sample was sufficiently dried under reduced pressure at $70^{\circ} \mathrm{C}$ for at least $48 \mathrm{~h}$. Solvents used were reagent grade of benzene, $o$-xylene, and toluene, and purified by the usual method before use.

Table I. Physical properties of branched low density polyethylenes ${ }^{\mathbf{a}}$

\begin{tabular}{|c|c|c|c|c|}
\hline \multirow{2}{*}{ Sample } & Density & \multirow{2}{*}{$\begin{array}{c}\bar{M}_{w} \times 10^{-4} \\
\quad(\mathrm{GPC})\end{array}$} & \multirow{2}{*}{$\begin{array}{c}\mathrm{CH}_{3} / 1000 \mathrm{C} \\
\text { (IR) }\end{array}$} & \multirow{2}{*}{$\frac{[\eta]}{100 \mathrm{~cm}^{3} \mathrm{~g}^{-1}}$} \\
\hline & $\mathrm{g} \mathrm{cm}^{-3}$ & & & \\
\hline BLDPE-1 & 0.921 & 8.7 & 26.5 & 0.967 \\
\hline BLDPE-2 & 0.921 & 9.1 & 23.6 & 1.022 \\
\hline BLDPE-3 & 0.924 & 8.8 & 20.7 & 0.780 \\
\hline BLDPE-4 & 0.917 & 17.5 & 30.4 & 1.070 \\
\hline BLDPE- 5 & 0.919 & 16.1 & 25.8 & 1.137 \\
\hline
\end{tabular}

a $\bar{M}_{w}$, weight-average molecular weight obtained by GPC; $\mathrm{CH}_{3} / 1000 \mathrm{C}$, the number of methyl groups per 1000 carbon atoms determined by infrared analysis; $[\eta]$, intrinsic viscosity measured in tetralin at $130^{\circ} \mathrm{C}$. 
Preparation Methods of Rapid and Slow Cooling Gels

A rapid cooling gel and a slow cooling gel were prepared in a glass tube of $c a .35 \mathrm{~cm}$ height and $1 \mathrm{~cm}$ diameter by the following procedure: An appropriate amount of sample and solvent together with a small steel ball of ca. $2 \mathrm{mg}$ weight $(0.8 \mathrm{~mm}$ diameter $)$ were placed in the glass tube and sealed. After the steel ball was fixed at the upper part of the glass tube by a magnet, the sample was dissolved completely in a solvent at an elevated temperature. Then, the rapid cooling gel was formed by dipping the glass tube quickly into cold water at $15^{\circ} \mathrm{C}$ for $3 \mathrm{~min}$. Finally, it was placed in a thermobath kept at $25^{\circ} \mathrm{C}$ for $18 \mathrm{~h}$. The slow cooling gel was formed by cooling the hot solution slowly till $25^{\circ} \mathrm{C}$ at a cooling rate of about $2^{\circ} \mathrm{Ch}^{-1}$ and maintained at $25^{\circ} \mathrm{C}$ for $18 \mathrm{~h}$. During the cooling process, the solution in a moderately concentrated region turned into a gel without precipitation.

\section{Measurement of Gel-Melting Temperature}

The gel-melting temperature was measured by the falling-ball (ball-drop) method pioneered by Takahashi and co-workers. ${ }^{7}$ The dependence of gel-melting temperature on the size of a steel ball was examined by a preliminary experiment, and that of a $2 \mathrm{mg}$ weight was selected. ${ }^{33}$ The steel ball was moved carefully to the surface of gel by magnetic manipulation and then the gel was warmed slowly at a heating rate of about $6^{\circ} \mathrm{Ch}^{-1}$, while the height of the steel ball from a reference level was recorded by a cathetometer as a function of temperature. By using the steel ball of $2 \mathrm{mg}$ weight, the gel-melting temperature could be measured precisely till the minimum polymer concentration $C^{*}$ required for gel formation from solution.

\section{Scanning Electron Microscope (SEM) and Polarizing Microscope}

Observation of crystallites was carried out using an Olympus Model BHA-P polarizing microscope and an Akashi Model ALPHA-10 scanning electron microscope. The specimen for SEM measurement was prepared as follows: After a gel was lyophilized, it was coated with a thin layer of gold by the vacuum evaporation technique.

\section{RESULTS AND DISCUSSION}

\section{Comparison of Critical Gelation Concentrations $C^{*}$ of Rapid Cooling and Slow Cooling Gels} Solutions of BLDPE in $o$-xylene, toluene, and benzene formed thermo-reversible gels in a moderately concentrated region, and turbidity of the gels increased with increasing polymer concentration. The turbidity of the gels shows that there are many crystallites in the gels. In a dilute solution region, crystalline precipitates separated from a solution without gel formation. It is speculated from these facts that a three-dimensional network of the gel is made up of crystallites. The minimum polymer concentration required for gel formation, i.e., the critical gelation concentration $C^{*}$, was determined experimentally by using a steel ball of $c a .2 \mathrm{mg}$ weight and the value of $C^{*}$ is summarized in Table II. The criterion of $C^{*}$ was taken as the concentration at which the steel ball placed on the surface of gel would no longer move downwards.

As shown in Table II, values of $C^{*}$ of the slow cooling gels are approximately $3 \mathrm{~g} /$ $100 \mathrm{~cm}^{3}$, while those of the rapid cooling gels are about $1 \mathrm{~g} / 100 \mathrm{~cm}^{3}$. The $C^{*}$ of the slow cooling gels are considerably higher than those of the rapid cooling gels, and it is obvious that the $C^{*}$ strongly depends on the cooling rate of a BLDPE solution. Recently, Mandelkern and co-workers $^{31}$ investigated the molecular weight dependence of the critical gelation concentration $C^{*}$ of linear polyethylene gels and derived experimentally the relationship, $C^{*}=k M^{-\alpha}$, where $k$ is a characteristic constant of a polymer-solvent system and $\alpha$ is a constant which varies from about 0.2 to 0.3 for the polyethylenes having molecular weight 
Table II. Values of $C^{*}$ and $C_{0}$ for branched low density polyethylenes ${ }^{\mathrm{a}}$

\begin{tabular}{|c|c|c|c|c|c|c|c|}
\hline \multirow{4}{*}{ Sample } & \multicolumn{4}{|c|}{$C^{*}$} & & & \multirow{4}{*}{$\frac{C_{0}}{\mathrm{~g} / 100 \mathrm{~cm}^{3}}$} \\
\hline & & & & & & & \\
\hline & \multicolumn{2}{|c|}{$o$-Xylene } & \multicolumn{2}{|c|}{ Toluene } & \multicolumn{2}{|c|}{ Benzene } & \\
\hline & (a) & (b) & (a) & (b) & (a) & (b) & \\
\hline BLDPE-1 & 1.31 & 2.90 & 1.15 & 2.81 & 0.97 & 2.98 & 0.71 \\
\hline BLDPE-2 & 1.41 & 2.85 & 1.25 & 2.90 & 1.01 & 2.95 & 0.69 \\
\hline BLDPE-3 & 1.70 & 2.95 & 1.45 & 2.96 & 1.35 & 3.01 & 0.71 \\
\hline BLDPE-4 & 0.80 & 2.60 & 0.71 & 2.70 & 0.81 & 2.95 & 0.50 \\
\hline BLDPE-5 & 0.85 & 2.65 & 0.79 & 2.75 & 1.11 & 2.97 & 0.53 \\
\hline
\end{tabular}

a $C^{*}$, the minimum polymer concentration at which gelation takes place (the critical gelation concentration); (a), rapid cooling gel; (b), slow cooling gel; $C_{0}$, the concentration at which polymer chains begin to overlap with each other in a solution, whose value was estimated by eq 1 .

range of $\bar{M}_{w}=0.3 \times 10^{4}-48.0 \times 10^{4}$. In a molecular weight range of the BLDPE samples studied here, the $C^{*}$ of rapid cooling gel in $o$-xylene or toluene depends slightly on the molecular weight as shown in Table II, while the molecular weight dependence of the $C^{*}$ of slow cooling one is little. In the lower concentration region than the $C^{*}$, crystalline phase separation took place with cooling for both rapid and slow cooling systems.

Comparison between the $C^{*}$ and concentration $C_{0}$ at which BLDPE chains begin to overlap with each other in a solution is one of the useful means for investigation of the gelation mechanism of the BLDPE from the solution. According to Klein, ${ }^{34}$ Takahashi, ${ }^{35}$ or Mandelkern, ${ }^{31}$ the concentration $C_{0}$ at which quasi-ideal polymer coils begin to overlap in a solution is given by assuming a statistical conformation

$$
C_{0}=\frac{3 M}{4 \pi\left\langle S^{2}\right\rangle^{3 / 2} N_{\mathrm{A}}}=K M^{-1 / 2}
$$

where $\left\langle S^{2}\right\rangle$ is the mean square radius of gyration of the polymer, $M$ is the molecular weight, $N_{\mathrm{A}}$ is Avogadro's number, and $K$ is a constant which depends upon the polymersolvent system. In order to estimate the $C_{0}$ of the present samples, the weight-average mo- lecular weight $\bar{M}_{w}$ was utilized for $M$ in eq 1, using $K=2.1$ which was estimated by Takahashi for polyethylene samples. ${ }^{35}$ Values of $C_{0}$ thus calculated are summarized in Table II. The data in this table suggest that the $C^{*}$ of both rapid and slow cooling gels are considerably higher than the $C_{0}$ for each samplesolvent system, and moreover the difference between the $C^{*}$ and the $C_{0}$ becomes larger in the case of slow cooling. Therefore, the gelation of a BLDPE solution requires a much higher concentration than the $C_{0}$.

\section{Morphology in the Vicinity of $C^{*}$}

The morphology of crystallite was observed above and below the critical gelation concentration $C^{*}$, and the dependence of crystalline structure on the condition for gel formation was investigated. Examples of photographs of crystallites in BLDPE-2-benzene systems at $0.7 \mathrm{~g} / 100 \mathrm{~cm}^{3}$, i.e., in the vicinity of $C_{0}$, where phase separation took place, are shown in Figure 1(a) for the rapid cooling and also in Figure 1(b) for the slow cooling. They were photographed by a polarizing microscope with crossed nicols. It is obvious from both photographs that the typical spherulitic pattern with a four-leaf clover as well as the Maltese cross can be observed, and the phase 
separation systems definitely include many spherulites. This spherulitic crystallization indicates that the crystallization occurs without any gelation even in the vicinity of $C_{0}$ where the overlap of polyethylene molecules begins. In this sense, as pointed out by Mandelkern, ${ }^{31}$ the molecular overlap is a necessary condition for crystallization, but it is not a sufficient one for gelation in the case of polyethylene. On the other hand, Tan and co-workers ${ }^{24}$ studied thermo-reversible gelation of noncrystallizable atactic polystyrene in $\mathrm{CS}_{2}$, and investigated the dependence of the critical gelation concentration $C^{*}$ on the molecular weight. Then, they reported that the $C^{*}$ of atactic polystyrene strongly depended on the molecular weight and moreover the $C^{*}$ was in good accordance with the $C_{0}$. From this viewpoint,

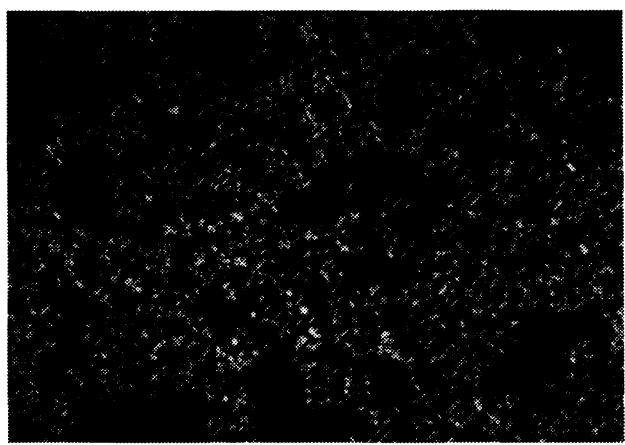

(a) by Rapid Cooling

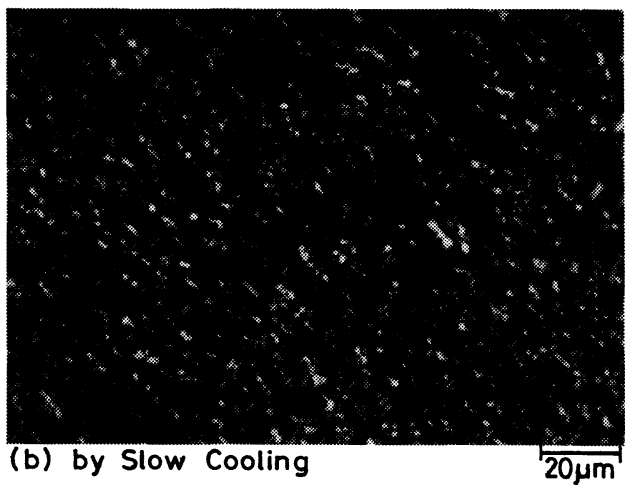

Figure 1. Polarizing micrographs of spherulites with crossed nicols for BLDPE-2-benzene systems at a concentration of $0.7 \mathrm{~g} / 100 \mathrm{~cm}^{3}$ where phase separation took place: (a), by rapid cooling; (b), by slow cooling. they proposed that the molecular overlap was a necessary condition for gelation of atactic polystyrene in $\mathrm{CS}_{2}$. Therefore, the gelation mechanism of BLDPE is considered to be different from that of atactic polystyrene.

On the other hand, Figure 2(a) and 2(b) are photographs of crystallites in BLDPE-2benzene systems at $3.5 \mathrm{~g} / 100 \mathrm{~cm}^{3}$, i.e., above the $C^{*}$, obtained by the rapid cooling and by the slow cooling, respectively. Both systems were converted to gels. In these photographs, many spherulites are also observed similarly as Figure 1. Comparing the spherulites in Figure 1 with those in Figure 2, the size of spherulites obtained from a higher concentration solution (in Figure 2) is considerably larger than that shown in Figure 1, and moreover the spherulites in both rapid and slow cooling gels (in

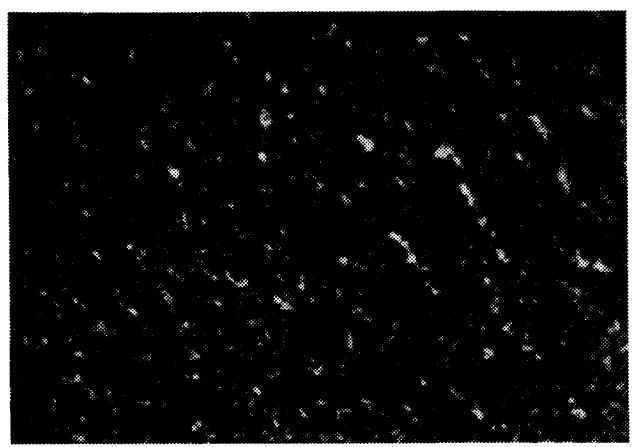

(a) by Rapid Cooling

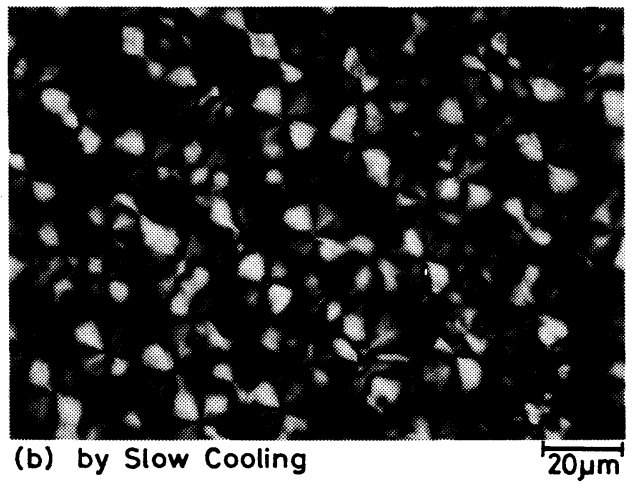

Figure 2. Polarizing micrographs of spherulites with crossed nicols for BLDPE-2-benzene systems at a concentration of $3.5 \mathrm{~g} / 100 \mathrm{~cm}^{3}$ where gelation took place: (a), by rapid cooling; (b), by slow cooling. 
Figure 2) are in contact with each other. Below the $C^{*}$ (in Figure 1), the spherulites are small and isolated from one another. These tendencies were similar to those observed in toluene and $o$-xylene systems and also to those for isotactic polypropylene in organic solvents reported previously. ${ }^{18}$ Therefore, in order to form a gel from a solution, it is necessary that the system is filled with a large number of spherulites which are in contact with each other. That is, contact of crystallites (spherulites) is conducive to gelation from at BLDPE solution.

\section{Morphology of Rapid and Slow Cooling Gels at Higher Concentration Region}

In the previous section, we showed that many spherulites existed in a gel, they were in contact with each other, and these spherulites formed a three-dimensional network structure of the gel. So, a more detailed morphology of the rapid and slow cooling gels was investigated at a higher concentration region.

The difference in the refractive indices between a BLDPE $\left(n_{\mathrm{D}}=1.50-1.53\right)^{36}$ and the solvents $\left(n_{\mathrm{D}}=1.49-1.51\right)^{36}$ is little, and thus the morphology could not be observed clearly under an ordinary microscope. So, the specimen was prepared by the following technique in order to enlarge the refractive index difference between the BLDPE and the solvent. After the gel was carefully placed on a slide glass of a microscope, the specimen was washed with ethanol $\left(n_{\mathrm{D}}=1.36\right)^{36}$ and a cover glass was put on the gel, and then the morphology was observed. Examples of photographs of BLDPE-2-benzene gels at $5 \mathrm{~g}$ / $100 \mathrm{~cm}^{3}$ are shown in Figure 3(a) for the rapid
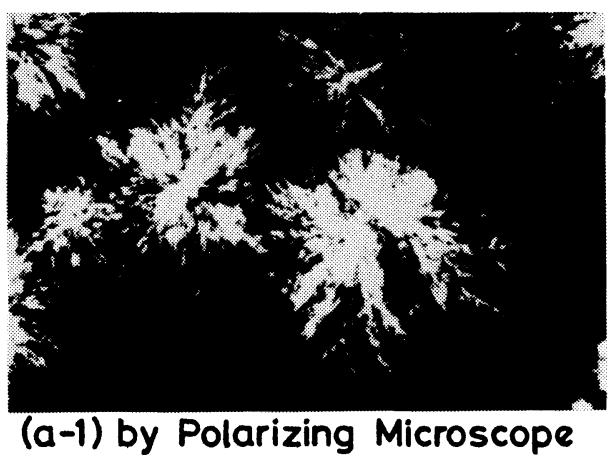

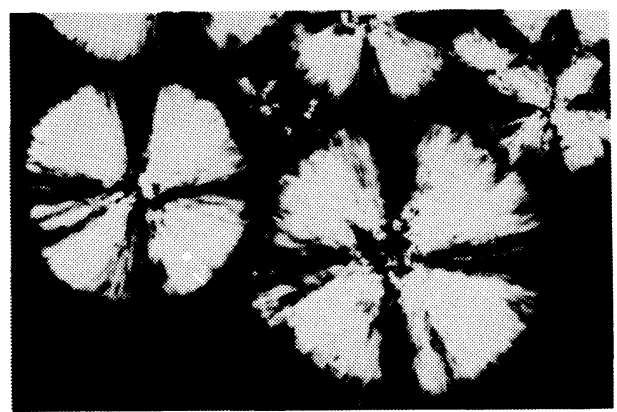

(b-1) by Polarizing Microscope
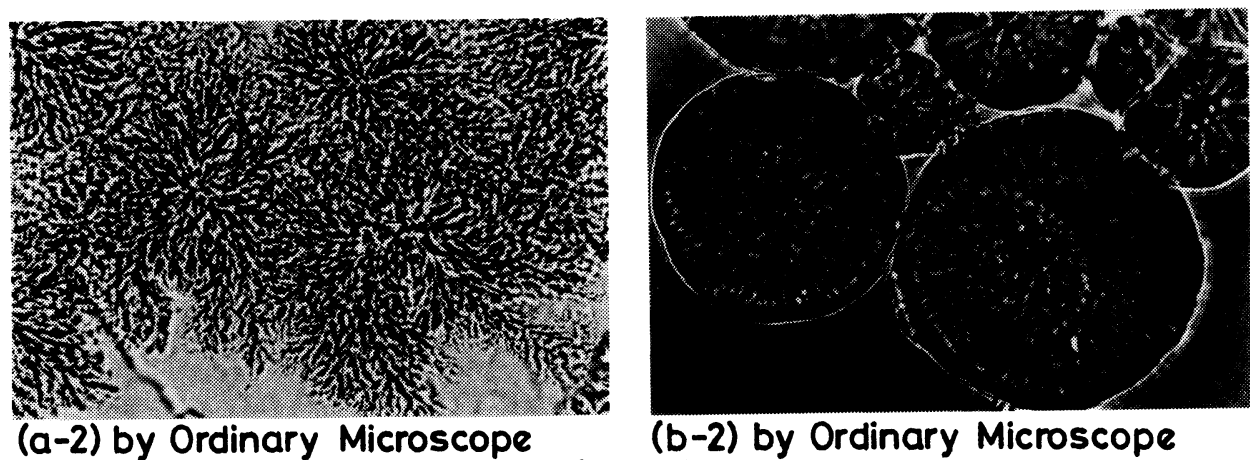

(b-2) by Ordinary Microscope $30 \mu \mathrm{m}$

Figure 3. Comparison of spherulites photographed by a polarizing and an ordinary microscopes for BLDPE-2-benzene gels at a concentration of $5 \mathrm{~g} / 100 \mathrm{~cm}^{3}:(\mathrm{a}-1)$ and (a-2), rapid cooling gel; (b-1) and (b-2), slow cooling gel. 
cooling and also in Figure 3(b) for the slow cooling. In this figure, (a-1) and (b-1) were photographed by a polarizing microscope with crossed nicols, while (a-2) and (b-2) were so by an ordinary microscope.

The size of spherulites in Figure 3 is remarkably larger than that shown in Figures 1 and 2. In Figure 3(a-1) and 3(a-2) which were photographed at the same specimen, many spherulites with imperfect spherical form are observed and they proceed to the radial direction from their nuclei. Moreover, tips of the spherulite (Figure 3(a-2)) grow like a branch of a tree such as a dendrite and they link with the tips of other spherulites, and thus the threedimensional network structure of the gel is

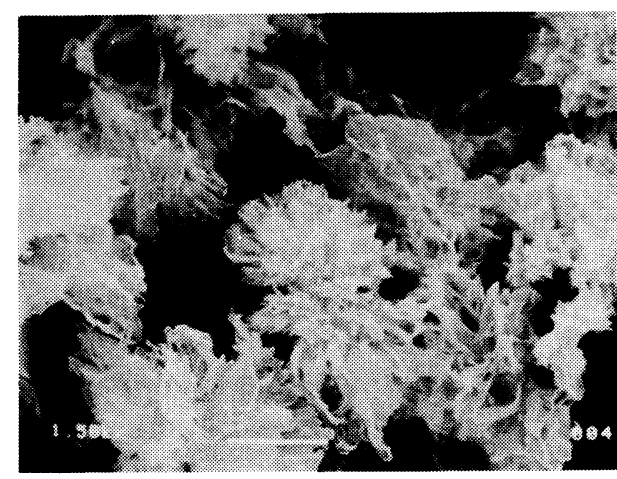

\section{(a-1) in Rapid Cooling Gel}

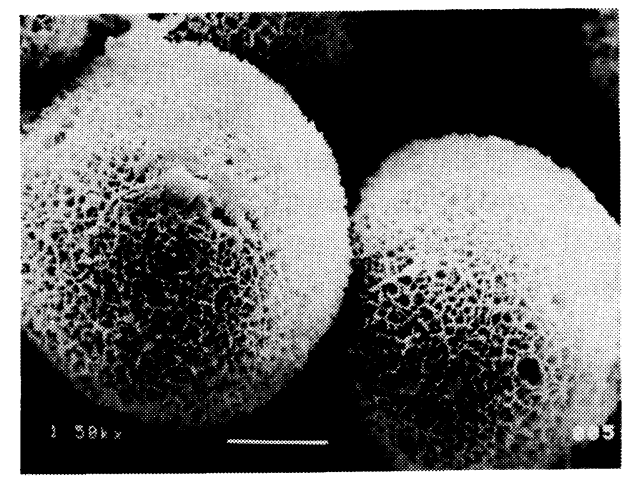

\section{$(b-1)$ in Slow Cooling Gel}

observed. On the other hand, in Figure 3(b-1) and $3(b-2)$, the spherulites with almost perfect spherical form are observed and the Maltese cross is much clearer in comparison with that shown in Figure 3(a-1). Moreover, the spherulites contact with each other, and they form the three-dimensional network structure of the gel.

We further investigated the spherulites in gels using SEM. The photographs are shown in Figure 4(a-1) and 4(a-2) for the rapid cooling gel and also in Figure 4(b-1) and 4(b-2) for the slow cooling one. In the figure, $(a-2)$ and (b-2) are close-up photographs of (a-1) and (b1), respectively. It is extremely obvious from Figure $4(a-1)$ and $4(a-2)$ that the imperfect
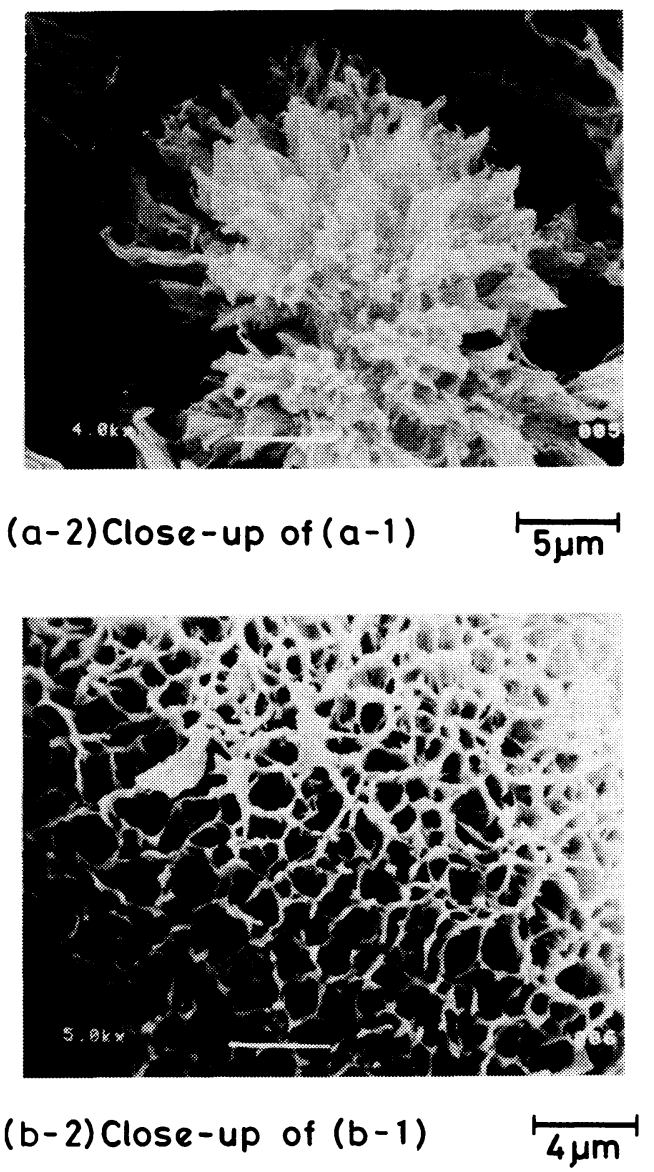

Figure 4. Comparison of spherulites photographed by SEM for BLDPE-2-benzene gels at a concentration of $5 \mathrm{~g} / 100 \mathrm{~cm}^{3}$ : (a-1) and (a-2), by rapid cooling; (b-1) and (b-2), by slow cooling. 


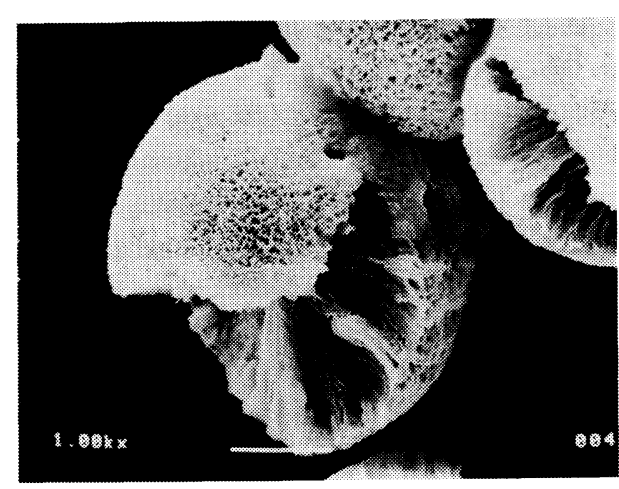

(A) Long Shot of Spherulite

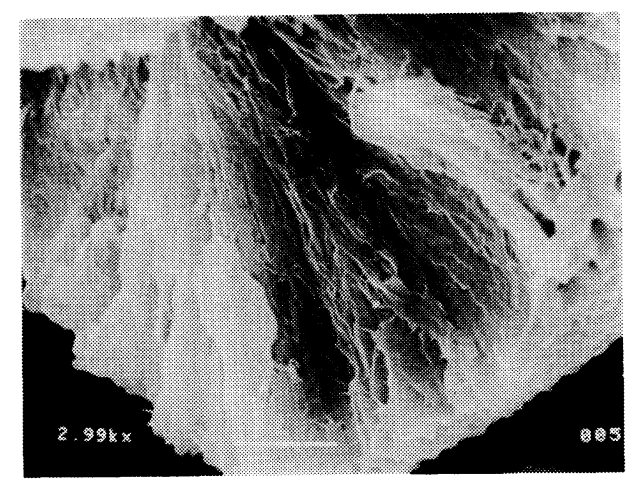

\section{(A') Close-up of Photo(A)}

Figure 5. Internal structure of spherulite crystallized by slow cooling procedure for BLDPE-2-benzene system at a concentration of $3 \mathrm{~g} / 100 \mathrm{~cm}^{3}$.

spherulites contacting with each other can be observed, and the surface is like a corallite.

On the other hand, in Figure $4(b-1)$, the spherulites with perfect spherical form are observed and they are in contact with each other. The diameter of the spherulite is approximately $30 \mu \mathrm{m}$ and the semblance is that of an aegagropila. It is considered, therefore, that the slow cooling procedure promotes the crystallization (growth of spherulite) and, as a result, the size of spherulite becomes larger and the semblance becomes almost perfect spherical form in the gel.

The internal structure of spherulite obtained by slow cooling is also shown in Figure 5, which was accidentally broken during the washing process with ethanol. In this figure, the lamellar structure can be observed inside the spherulite and the development of lamella proceeds to the radial direction from the nucleus.

\section{Mechanism of Gelation and Structure of Gel}

In the above sections, we investigated the morphological difference of spherulites in gels obtained by the rapid cooling and the slow cooling in a wide range of polymer concentrations, and showed that the semblance of spherulite as well as the critical gelation concentration $C^{*}$ strongly depended on the cooling rate of a solution. For both rapid and slow cooling procedures, the size as well as the number of spherulite increased gradually with increasing polymer concentration and, by and by, the spherulites in a moderately concentrated region (higher than about $3.5 \mathrm{~g} / 100 \mathrm{~cm}^{3}$ ) became an appropriate size so as to contact with each other. At this time, it is considered that a system is filled with spherulites and consequently crosslinking junction points and the three-dimensional network structure may be formed through contact of a large number of spherulites. As a result, the system converts to a gel without separation of the spherulites from a solution, confining the solution in this crystalline network. Particularly in the case of the slow cooling procedure, the abovementioned speculation for the gelation mechanism is strongly supported from the fact that the molecular weight dependence of the critical gelation concentration $C^{*}$ of a slow cooling gel is little.

In a rapid cooling gel, however, the $C^{*}$ depended slightly on the molecular weight, i.e., the $C^{*}$ decreased with increasing molecular weight (see Table II). Moreover, from the microscopic observation, the rapid cooling system in the vicinity of $C^{*}$ has already included spherulites though they are isolated from one another, while the spherulites have 
contacted with each other at least at a concentration of $3.5 \mathrm{~g} / 100 \mathrm{~cm}^{3}$ (see Figure 2(a)), indicating that the gelation of a BLDPE solution with concentration less than about $3.5 \mathrm{~g} /$ $100 \mathrm{~cm}^{3}$ takes place without contact of the spherulites. In the case of the rapid cooling system in a concentration region from the $C^{*}$ to about $3 \mathrm{~g} / 100 \mathrm{~cm}^{3}$, where no gelation occurs by the slow cooling, it is predicted that the gelation takes place by contact or intertwinement of polyethylene molecules which are confined among the spherulites crystallized partially in the system when the solution is cooled rapidly.

\section{Determination of Gel-Melting Temperature}

An example of measurement of gel-melting temperature by the falling-ball method is illustrated in Figure 6. The temperature at which the horizontal and vertical straight lines intersected was taken as the gel-melting temperature $T_{\mathrm{m}}^{\mathrm{g}}$. The measurements were repeated several times at each concentration, and the reproducibility of the data was within about $\pm 1^{\circ} \mathrm{C}$ for both rapid and slow cooling gels. The average was used for subsequent discussion.

\section{Dependence of Gel-Melting Temperature on \\ Polymer Concentration, Branching Degree, and Molecular Weight \\ Examples of the relation between gel-melt-} ing temperature $T_{\mathrm{m}}^{\mathrm{g}}\left({ }^{\circ} \mathrm{C}\right)$ and polymer concentration $C\left(\mathrm{~g} / 100 \mathrm{~cm}^{3}\right)$ are shown in Figure 7(a) for the rapid cooling gels and also in Figure 7(b) for the slow cooling gels in benzene system. In this figure, a phase separation region is also illustrated, and it is very obvious that the critical gelation concentration $C^{*}$ of the slow cooling gels is much higher than those of the rapid cooling gels. The difference in $T_{\mathrm{m}}^{\mathrm{g}}$ between the rapid and slow cooling gels for a same sample was little. In either Figure 7(a) or 7(b), the $T_{\mathrm{m}}^{\mathrm{g}}$ of each sample increases slowly with increasing polymer concentration.

In samples having almost similar weight-

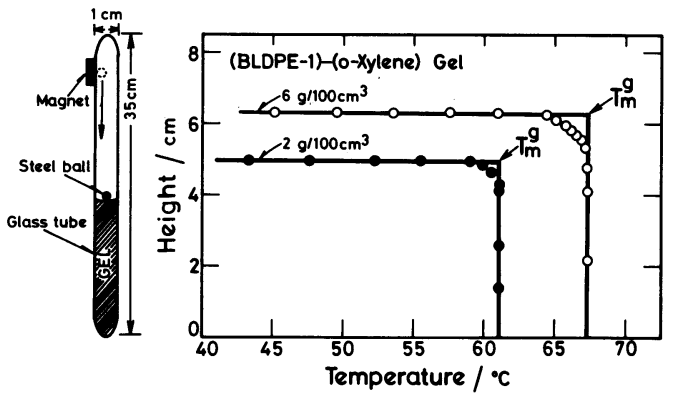

Figure 6. Schematic drawing of an apparatus for gelmelting temperature and examples of measurements of gel-melting temperature by the falling-ball method.

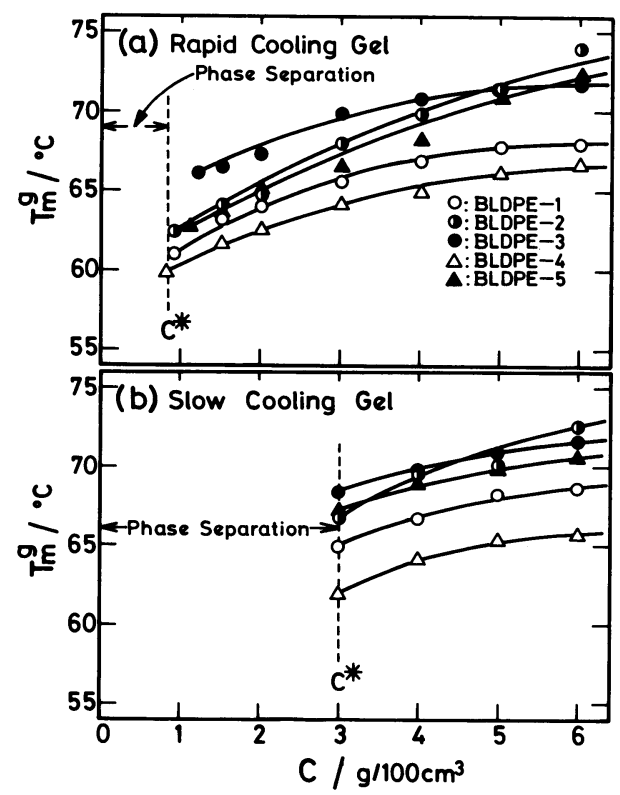

Figure 7. Relation between gel-melting temperature $T_{\mathrm{m}}^{\mathrm{g}}\left({ }^{\circ} \mathrm{C}\right)$ and polymer concentration $C\left(\mathrm{~g} / 100 \mathrm{~cm}^{3}\right)$ for BLDPE-benzene gels: (a), rapid cooling gel; (b), slow cooling gel. $C^{*}$ shows the critical gelation concentration.

average molecular weights, i.e., in BLDPE-13 or in BLDPE-4 -5 , the $T_{\mathrm{m}}^{\mathrm{g}}$ decreases with increasing branching degree $\left(\mathrm{CH}_{3} / 1000 \mathrm{C}\right)$. Moreover, in samples having similar values of $\mathrm{CH}_{3} / 1000 \mathrm{C}$, i.e., in BLDPE-1 and BLDPE-5, the $T_{\mathrm{m}}^{\mathrm{g}}$ of BLDPE-5 having large molecular weight $\bar{M}_{w}$ is higher than that of BLDPE-1. Similar tendencies were also shown for other solvent systems. Consequently, it is found that the gel-melting temperature depends on poly- 


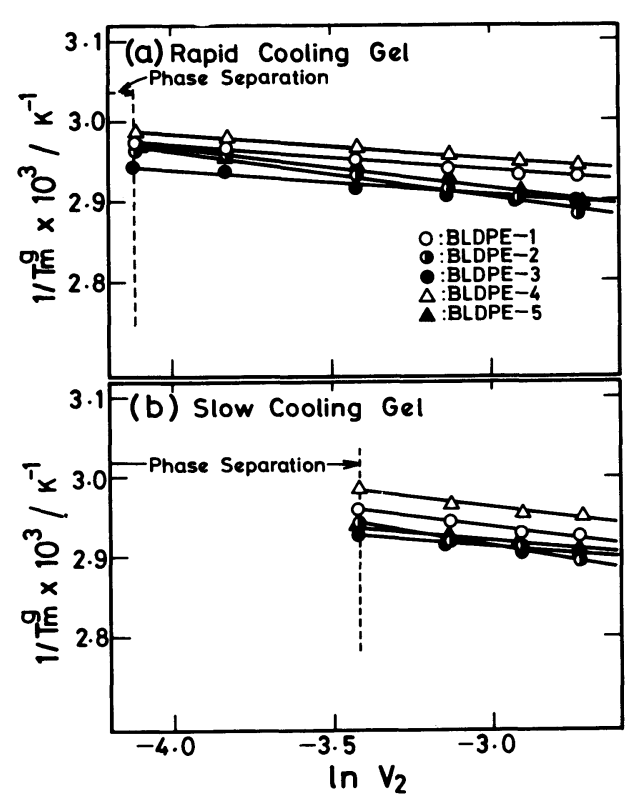

Figure 8. Relation between the reciprocal absolute gelmelting temperature $1 / T_{\mathrm{m}}^{\mathrm{g}}\left(\mathrm{K}^{-1}\right)$ and logarithm of the volume fraction $\ln V_{2}$ of BLDPE in a gel according to eq 2. This figure was constructed using the data shown in Figure 7.

mer concentration, branching degree, and molecular weight. The $T_{\mathrm{m}}^{\mathrm{g}}$ increases with increasing polymer concentration as well as molecular weight, but decreases with increasing branching degree.

Eldridge and Ferry derived empirically the following relation between the absolute gelmelting temperature $T_{\mathrm{m}}^{\mathrm{g}}(\mathrm{K})$ and the volume fraction of polymer $V_{2}$ in a gel $^{37}$.

$$
\ln V_{2} \doteq \text { const. }+\Delta H_{\mathrm{m}} / R T_{\mathrm{m}}^{\mathrm{g}}
$$

where $\Delta H_{\mathrm{m}}$ is the heat absorbed on formation of one mol of junction points and $R$ is the gas constant. It has been widely recognized that the gel-melting temperatures of a large number of natural polymer or synthetic polymer gels obey this empirical relation. So, the applicability of eq 2 was examined for the present data. In this study, it is not the purpose to estimate the value of $\Delta H_{\mathrm{m}}$. According to eq 2, a plot of $\ln V_{2}$ vs. $1 / T_{\mathrm{m}}^{\mathrm{g}}\left(\mathrm{K}^{-1}\right)$ should yield a straight line. The plots are shown in Figure 8(a) and 8(b) for the rapid cooling gel and for the slow cooling one in benzene system, respectively. In either Figure 8(a) or 8(b), good straight lines are obtained for all samples. Similar relations were also obtained in $o$ xylene and toluene systems. Consequently, the concentration dependence of gel-melting temperature can be represented well by eq 2 .

\section{CONCLUSIONS}

The following results were obtained for the structure and the morphology of BLDPE gels prepared by rapid and slow cooling procedures from solutions:

1. A BLDPE in toluene, benzene, and $o$ xylene converts to a gel from a moderately concentrated solution on cooling, which in turn converts to a solution on heating. The solgel transition is thermo-reversible.

2. The critical gelation concentrations $C^{*}$ of both rapid and slow cooling gels are remarkably higher than the concentration $C_{0}$ at which polymer chains begin to overlap with each other in a solution and the gelation takes place after the crystallization has already occurred. The molecular overlap as well as the crystallization are a necessary conditions for the gelation.

3. The $C^{*}$ depends considerably on the cooling rate of a solution: The $C^{*}$ of rapid cooling gels are about $1 \mathrm{~g} / 100 \mathrm{~cm}^{3}$, which depend slightly on the molecular weight, i.e., the $C^{*}$ decreases with increasing molecular weight, indicating that the gelation in the vicinity of $C^{*}$ takes place by the molecular overlap or intertwinement of polymer chains confined among the spherulites crystallized partially in a system. The $C^{*}$ of slow cooling gels are about $3 \mathrm{~g} / 100 \mathrm{~cm}^{3}$ regardless of the difference in molecular weight, indicating that the gelation takes place by the contact of spherulites.

4. A large number of spherulites are observed in both rapid and slow cooling gels, but the semblance of spherulites depends consid- 
erably on the cooling rate of a solution: The spherulite obtained by the rapid cooling is imperfect spherical form (dendrite), while that by the slow cooling is almost perfect spherical form (typical spherulite).

5. The difference in gel-melting temperatures $T_{\mathrm{m}}^{\mathrm{g}}$ between the rapid cooling gel and the slow cooling one for a same sample is little. The $T_{\mathrm{m}}^{\mathrm{g}}$ depends on polymer concentration, molecular weight, and branching degree: The $T_{\mathrm{m}}^{\mathrm{g}}$ increases with increasing polymer concentration as well as molecular weight, but decreases with increasing branching degree. The concentration dependence of $T_{\mathrm{m}}^{\mathrm{g}}$ can be represented well by the Eldridge-Ferry's type of plot: The reciprocal absolute gel-melting temperature $1 / T_{\mathrm{m}}^{\mathrm{g}}\left(\mathrm{K}^{-1}\right)$ has a linear relationship to the logarithm of volume fraction $\ln V_{2}$ of BLDPE in the gel.

Acknowledgement. The authors wish to express their sincere appreciation to $\mathrm{Mr}$. Yoshihiro Motegi of the Ohita Laboratory, Showa Denko Co. for supplying and characterizing the BLDPE samples.

\section{REFERENCES}

1. A. Takahashi, T. Nakamura, and I. Kagawa, Polym. J., 3, 207 (1972).

2. A. Takahashi, Polym. J., 4, 379 (1973).

3. A. Takahashi and S. Hiramitsu, Polym. J., 6, 103 (1974).

4. T. Kato, M. Yokoyama, and A. Takahashi, Colloid Polym. Sci., 256, 15 (1978).

5. R. Benson, J. Maxfield, D. E. Axelson, and L. Mandelkern, J. Polym. Sci., Polym. Phys. Ed., 16, 1583 (1978).

6. A. Takahashi, T. Kawaharada, and T. Kato, Polym. J., 11, 671 (1979).

7. A. Takahashi, M. Sakai, and T. Kato, Polym. J., 12, 335 (1980).

8. C. O. Edwards and L. Mandelkern, J. Polym. Sci., Polym. Lett. Ed., 20, 355 (1980).

9. P. J. Flory, "Principles of Polymer Chemistry," Cornell Univ. Press, Ithaca, N. Y., 1953, Chapter IX.

10. P. G. de Gennes, "Scaling Concepts in Polymer Physics," Cornell Univ. Press, Ithaca, N. Y., 1979,
Part A (Chapter V).

11. H. Matsuda, K. Araki, M. Yamada, and S. Kuroiwa, Rep. Prog. Polym. Phys. Jpn., 20, 69 (1977).

12. H. Matsuda, K. Araki, H. Fujimatsu, and S. Kuroiwa, Polym. J., 13, 587 (1981).

13. H. Matsuda, H. Fujimatsu, M. Imaizumi, and S. Kuroiwa, Polym. J., 13, 807 (1981).

14. H. Matsuda, M. Imaizumi, H. Fujimatsu, S. Kuroiwa, and M. Okabe, Polym. J., 16, 151 (1984).

15. M. Okabe, M. Isayama, and H. Matsuda, Polym. J., 17, 369 (1985).

16. M. Okabe and H. Matsuda, Kobunshi Ronbunshu, 42, 397 (1985).

17. M. Okabe, M. Isayama, and H. Matsuda, J. Appl. Polym. Sci., 30, 4735 (1985).

18. H. Matsuda, T. Inoue, M. Okabe, and T. Ukaji, Polym. J., 19, 323 (1987).

19. A. Labudzinska and A. Ziabicki, Kolloid Z.-Z. Polym., 243, 21 (1971).

20. E. Pines and W. Prins, Macromolecules, 6, 888 (1973).

21. G. T. Feke and W. Prins, Macromolecules, 7, 527 (1974).

22. D. R. Paul, J. Appl. Polym. Sci., 11, 439 (1976).

23. S. Wellinghoff, J. Shaw, and E. Baer, Macromolecules, 12, 932 (1979).

24. H.-M. Tan, A. Moet, A. Hiltner, and E. Baer, Macromolecules, 16, 28 (1983).

25. M. Komatsu, T. Inoue, and K. Miyasaka, Polym. Prepr. Jpn., 33, 2327 (1984).

26. M. Komatsu, T. Inoue, and K. Miyasaka, Sen-i Gakkaishi, 40, 316 (1984).

27. J.-M. Guenet, B. Lotz, and J.-C. Wittmann, Macromolecules, 18, 420 (1985).

28. R. F. Boyer, E. Baer, and A. Hiltner, Macromolecules, 18, 427 (1985).

29. M. Komatsu, T. Inoue, and K. Miyasaka, J. Polym. Sci., Polym. Phys. Ed., 24, 303 (1986).

30. K. Kawanishi, Y. Takeda, and T. Inoue, Polym. J., 18, 411 (1986).

31. R. C. Domszy, R. Alamo, C. O. Edwards, and L. Mandelkern, Macromolecules, 19, 310 (1986).

32. A. Takahashi and T. Kato, Res. Rep. Fac. Eng., Mie Univ., 1, 97 (1976).

33. M. Okabe, K. Muto, and H. Matsuda, Polym. Prepr. Jpn., 33, 936 (1984); M. Okabe, K. Muto, and H. Matsuda, ibid., 34, 1026 (1985).

34. J. Klein, Macromolecules, 11, 852 (1978).

35. M. Kawaguchi, S. Kubota, and A. Takahashi, Polym. Prepr. Jpn., 32, 1659 (1983).

36. The Chemical Society of Japan, Ed., "Kagaku Binran," Maruzen, Tokyo, 1984, p I-561 and p 1111.

37. J. E. Eldridge and J. D. Ferry, J. Phys. Chem., 58, 992 (1954). 